\title{
Weak and Strong Bitopological Lindel of Space
}

\section{K.A Venkatesh* \& S. Balasubramaniant}

\begin{abstract}
Keywords: Topological space, bitopological space, continuous function and Lindelof space. This paper deals with structural properties of weak(strong) Lindelof Bitopological space.
\end{abstract}

\section{Introduction}

J.C. Kelly[1] introduced the concept of bitopological space. S. Balasubramanian and G. Koteswara Rao[3] introduced the concept of strong (weak) Lindelof bitopology space. In this paper, we define strong (weak) continuous function on bitopological space and study the structural properties of Lindelof bitopological space.

\section{Definition}

Definition 2.1[1] : A non empty set $X$ together with two topologies $\Gamma, \& \Gamma_{2^{\prime}}$ denoted by $\left(X, \Gamma_{1}, \Gamma_{2}\right)$ is called bitopological space.

Definition 2.2[2] : A bitopological space $\left(X, \Gamma_{1}, \Gamma_{2}\right)$ is said to be a compact space if $X$ is $\Gamma_{1}$-compact and $\Gamma_{2}$-compact.

* Co-ordinator, Dept. of MCA, Alliance Business Academy, BTM II statge, Bangalore - 560076

+ Reader, PG Dept. of Mathematics, SS\&N College, Narasaraopet, Andra Pradesh. 
Definition 2.3[3] : A bitopological space $\left(X, \Gamma_{1}, \Gamma_{2}\right)$ is scid to be strong Lindelof bitopological space if both $\left(X, \Gamma_{1}\right)$ and $\left(X, \Gamma_{2}\right)$ are Lindelof.

Definition 2.4[3] : A bitopological space $\left(X, \Gamma_{1}, \Gamma_{2}\right)$ is said to be weak Lindelof bitopological space if either $\left(X, \Gamma_{1}\right)$ or $\left(X, \Gamma_{2}\right)$ is Lindelof.

Definition 2.5: Let $\left(X, \Gamma_{1}, \Gamma_{2}\right)$ and $\left(Y, \sigma_{1}, \sigma_{2}\right)$ be any two bitopological spaces and $f: \rightarrow Y$ be a single valued function, $f$ is said to be

(a) weak continuous if either

$$
f:\left(X, \Gamma_{1}\right) \rightarrow\left(\gamma, \sigma_{1}\right) \text { or } f:\left(X, \Gamma_{2}\right) \rightarrow\left(Y, \sigma_{2}\right) \text { is continuous. }
$$

(b) Strong continuous if both

$$
f:\left(X, \Gamma_{1}\right) \rightarrow\left(\gamma, \sigma_{1}\right) \text { and } f:\left(X, \Gamma_{2}\right) \rightarrow\left(\gamma, \sigma_{2}\right) \text { are continuous. }
$$

Definition 2.6: Let $\left(X, \Gamma_{1}, \Gamma_{2}\right)$ and $\left(Y, \sigma_{1}, \sigma_{2}\right)$ be any two bitopological spaces and $f: x \rightarrow y$ be a single valued function, $f$ is said to be

(c) weak open if either

$$
f:\left(X, \Gamma_{1}\right) \rightarrow\left(Y, \sigma_{1}\right) \text { or } f:\left(X, \Gamma_{2}\right) \rightarrow\left(Y, \sigma_{2}\right) \text { is open. }
$$

(d) Strong open if both

$$
f:\left(X, \Gamma_{1}\right) \rightarrow\left(Y, \sigma_{1}\right) \text { and } f:\left(X, \Gamma_{2}\right) \rightarrow\left(Y, \sigma_{2}\right) \text { are open. }
$$

\section{Main Results(Structural Properties):}

Theorem 3.1: (Weak) Strong continuous image of (weak) strong Lindelof bitological space is weak (strong) Lindelof.

\section{Proof:}

- Let $f:\left(X, \Gamma_{1}, \Gamma_{2}\right) \rightarrow\left(Y, \sigma_{1}, \sigma_{2}\right)$ be a strong continuous functions, where $\left(X, \Gamma_{1}\right.$, $\left.\Gamma_{2}\right)$ and $\left(Y, \sigma_{1}, \sigma_{2}\right.$ ) are two bitopological spaces. Let $y \in Y$ be an element such that $f^{-1}(y) \in X$. Put $x=f^{-1}(y)$, for ${ }^{f-1}(y) \in X$. since $X$ is strong Lindel of there exists $\Gamma 1$ - open set $U$ containing $f^{-1}(y)$ and,$\Gamma_{2}$-open set $V$ containing $f^{-1}(y)$ and hence $y \in f(U)$ and $y \in f(M)$ are open sets in sl and $\sigma_{2}$ respectively. Since $X$ is strong Lindelof and $f$ - is continuous, we have a countable collection of open sets $U$ and $V$ with respect to, $\Gamma_{1} \& \Gamma_{2}$ covering $X$ and countable collection of open sets $f(U)$ and $f\left(M\right.$ covering $f(X)$ with respect to $\sigma_{1} \& \sigma_{2}$ respectively. 
Hence the proof.

\section{Theorem 3.2}

If $(X, \Gamma 1, \Gamma 2)$ is weak (strong) Lindelof and $(Y, \sigma 1, \sigma 2)$ is compact then $X \times Y$ is weak(strong) Lindelof.

\section{Proof:}

From the definitions of Lindelofness, and the compactness, the result is obvious.

\section{Theorem 3.3:}

If an arbitrary product of weak(strong) Lindelof bitoplolgical space is Lindelof then

(i) Each component space is weak(strong) Lindelof

(ii) All but finitely many of the compact spaces is weak (strong) Lindelof.

\section{Proof:}

Let $x=p x_{\alpha \in 1}$ be strong Lindelof bitopological space. Since the projection maps are continuous and open,

(i) follows from theorem 3.2. For part (ii), let $x \hat{\imath} X$ then $x=(x)_{0} \hat{\imath} p_{\text {oll }} X_{0}$. Then there exists a finite subset $J$ of $I$ and open set $U_{b}$ in $X_{b}$ (bîj) such that $x \hat{I} p_{b i \hat{s}} U_{b}$ $x p_{\text {ofl-s }} X_{0}$ contained in $W$ from which, again by theorem 3.2, if follows that $X_{0}$ is Lindelof for aîl-J

\section{References}

[1] J. C. Kelly, Proc. London Maths., Soc. (3) (1962), 71.89.

[2] Rupa Bandyopadhaya \& A.P. Bornab Bull., Cal. Maths., Sco. 87, 29-38 (1995).

[3] S. Balasubramanian \& G. Koteswara Rao, Weak and Strong - Lindelof Bitopological spaces (Communicated). 